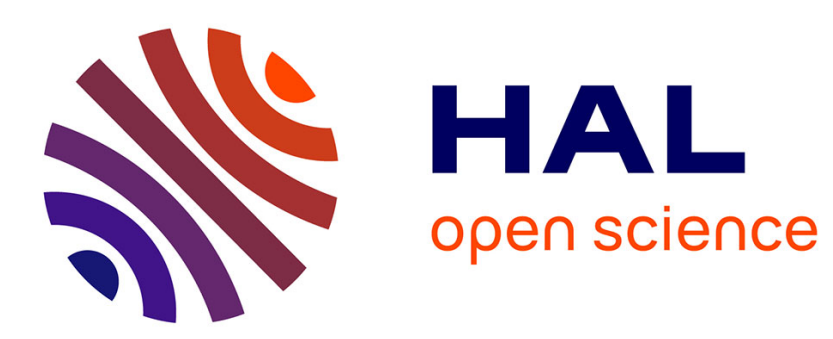

\title{
Spinors and isometric immersions of surfaces in 4-dimensional products
}

Julien Roth

\section{To cite this version:}

Julien Roth. Spinors and isometric immersions of surfaces in 4-dimensional products. Bulletin of the Belgian Mathematical Society - Simon Stevin, 2014, 21 (4), pp.635-652. hal-00933532

\section{HAL Id: hal-00933532}

\section{https://hal.science/hal-00933532}

Submitted on 20 Jan 2014

HAL is a multi-disciplinary open access archive for the deposit and dissemination of scientific research documents, whether they are published or not. The documents may come from teaching and research institutions in France or abroad, or from public or private research centers.
L'archive ouverte pluridisciplinaire HAL, est destinée au dépôt et à la diffusion de documents scientifiques de niveau recherche, publiés ou non, émanant des établissements d'enseignement et de recherche français ou étrangers, des laboratoires publics ou privés. 


\title{
SPINORS AND ISOMETRIC IMMERSIONS OF SURFACES IN 4-DIMENSIONAL PRODUCTS
}

\author{
JULIEN ROTH
}

\begin{abstract}
We prove a spinorial characterization of surfaces isometrically immersed into the 4-dimensional product spaces $\mathbb{M}^{3}(c) \times \mathbb{R}$ and $\mathbb{M}^{2}(c) \times \mathbb{R}^{2}$, where $\mathbb{M}^{n}(c)$ is the $n$ dimensional real space form of curvature $c$.
\end{abstract}

\section{INTRODUCTION}

In [4], Friedrich gave a spinorial characterization of surfaces in Euclidean 3-space. Namely, he proved that the existence of a so-called generalized Killing spinor $\psi$ on surface $\left(M^{2}, g\right)$, that is

$$
\nabla_{X} \psi=A(X) \cdot \psi,
$$

where $A$ is a symmetric $(1,1)$-tensor, is equivalent to the Gauss and Codazzi equations and therefore to an isometric immersion of $\left(M^{2}, g\right)$ into $\mathbb{R}^{3}$ with $-2 A$ as shape operator. Later on, Morel generalized in [9] this result for surfaces of the sphere $\mathbb{S}^{3}$ and the hyperbolic space $\mathbb{H}^{3}$ and we give in [12] an analogue for 3-dimensional homogeneous manifolds with 4-dimensional isometry group, as well as for surfaces into pseudo-Riemannian space forms [6] and Lorentzian products [13]. In a more recent work [2], we studied with Bayard and Lawn the spinorial characterization of surfaces into 4-dimensional space forms. A similar result was proved by Bayard for spacelike surfaces into the 4-dimensional Minkowski space [1].

In this paper, we extend this spinorial characterization for surfaces in the product spaces $\mathbb{M}^{3}(c) \times \mathbb{R}$ and $\mathbb{M}^{2}(c) \times \mathbb{R}^{2}$, where $\mathbb{M}^{n}(c)$ is the $n$-dimensional real space form of constant sectional curvature $c \neq 0$.

First we characterize immersions of surfaces into these product spaces by the existence of special spinor fields satisfying an appropriate generalized Killing-type equation, that is an equation involving the spinorial connection (see Theorem 3.1). Then, we show that this equation is equivalent to the corresponding Dirac equation with an additional condition on the norm of the spinor field (see Proposition 4.1 and Corollary 4.2).

\section{Preliminaries}

In this section of preliminaries, we will first recall some basics about surfaces into the product spaces $\mathbb{M}^{2}(c) \times \mathbb{R}^{2}$ and $\mathbb{M}^{3}(c) \times \mathbb{R}$. In particular, we will recall the compatibility equations assuring that a surface is isometrically immersed into one of these spaces. Then, we will give some facts about restrictions of spinors on a surface into a 4-dimensional space and deduce the particular spinor fields with which we will work in the sequel.

2010 Mathematics Subject Classification. 53C27, 53C42.

Key words and phrases. Surfaces, Dirac Operator, Isometric Immersions. 
2.1. Compatibilty equations. Let $\left(M^{2}, g\right)$ be a Riemannian surface isometrically immersed into the product space $P=\mathbb{M}^{2}(c) \times \mathbb{R}^{2}$ or $\mathbb{M}^{3}(c) \times \mathbb{R}$, endowed with the product metric $\widetilde{g}$. We denote by $F$ product structure of $P$. The map $F: T P \longrightarrow T P$ is defined by $F\left(X_{1}+X_{2}\right)=X_{1}-X_{2}$, where $X_{1}$ belongs to the first factor $\left(T \mathbb{M}^{2}(c)\right.$ or $\left.T \mathbb{M}^{3}(c)\right)$ and $X_{2}$ belongs to the second factor $\left(T \mathbb{R}^{2}\right.$ or $\left.T \mathbb{R}\right)$. Obviously, $F$ satisfies

$$
\begin{gathered}
F^{2}=I d \quad(\text { and } F \neq I d), \\
\widetilde{g}(F X, Y)=\widetilde{g}(X, F Y), \\
\widetilde{\nabla} F=0 .
\end{gathered}
$$

Moreover, we recall that the curvature of $(P, \widetilde{g})$ is given by

$$
\begin{aligned}
\widetilde{R}(X, Y) Z= & \frac{c}{4}[\langle Y, Z\rangle X-\langle X, Z\rangle Y+\langle F Y, Z\rangle F X-\langle F X, Z\rangle F Y \\
& +\langle Y, Z\rangle F X-\langle X, Z\rangle F Y+\langle Y, F Z\rangle X-\langle X, F Z\rangle Y]
\end{aligned}
$$

This product structure $F$ induces the existence of the following four operators

$$
f: T M \longrightarrow T M, h: T M \longrightarrow N M, s: N M \longrightarrow T M \text { and } t: N M \longrightarrow N M
$$

defined for any $X \in T M$ and $\xi \in N M$ by

$$
F X=f X+h X \quad \text { and } \quad F \xi=s \xi+t \xi .
$$

From Equations (1) and (2), $f$ and $t$ are symmetric and we have the following relations between these four operators

$$
\begin{aligned}
& f^{2} X=X-s h X, \\
& t^{2} \xi=\xi-h s \xi, \\
& f s \xi+s t \xi=0, \\
& h f X+t h X=0, \\
& \widetilde{g}(h X, \xi)=\widetilde{g}(X, s \xi),
\end{aligned}
$$

for any $X \in \Gamma(T M)$ and $\xi \in \Gamma(N M)$. Moreover, from Equation (3), we have

$$
\begin{aligned}
& \left(\nabla_{X} f\right) Y=S_{h Y} X+s(B(X, Y)), \\
& \nabla_{X}^{\perp}(h Y)-h\left(\nabla_{X} Y\right)=t(B(X, Y))-B(X, f Y), \\
& \nabla^{\perp}(t \xi)-t\left(\nabla_{X}^{\perp} \xi\right)=-B(s \xi, X)-h\left(S_{\xi} X\right), \\
& \nabla_{X}(s \xi)-s\left(\nabla_{X}^{\perp} \xi\right)=-f\left(S_{\xi} X\right)+S_{t \xi} X,
\end{aligned}
$$

where $B: T M \times T M \longrightarrow N M$ is the second fundamental form and for any $\xi \in T M, S_{\xi}$ is the Weingarten operator associated with $\xi$ and defined by $\widetilde{g}\left(S_{\xi} X, Y\right)=\widetilde{g}(B(X, Y), \xi)$ for any vectors $X, Y$ tangent to $M$.

Finally, from (4), we deduce that the Gauss, Codazzi and Ricci equations are respectively given by

$$
\begin{aligned}
R(X, Y) Z= & \frac{c}{4}[\langle Y, Z\rangle X-\langle X, Z\rangle Y+\langle f Y, Z\rangle f X-\langle f X, Z\rangle f Y \\
& \langle Y, Z\rangle f X-\langle X, Z\rangle f Y+\langle Y, f Z\rangle X-\langle X, f Z\rangle Y] \\
& +S_{B(Y, Z)} X-S_{B(X, Z)} Y
\end{aligned}
$$




$$
\begin{aligned}
\left(\nabla_{X} B\right)(Y, Z)-\left(\nabla_{Y} B\right)(X, Z)= & \frac{c}{4}[\langle f Y, Z\rangle h X-\langle f X, Z\rangle h Y \\
& +\langle Y, Z\rangle h X-\langle X, Z\rangle h Y]
\end{aligned}
$$

(17) $R^{\perp}(X, Y) \xi=\frac{c}{4}[\langle h Y, \xi\rangle h X-\langle h X, \xi\rangle h Y]+B\left(S_{\xi} Y, X\right)-B\left(S_{\xi} X, Y\right)$.

Conversely, let $\left(M^{2}, g\right)$ a Riemannian surface endowed with a rank 2 vector bundle $E$ endowed with a metric and a compatible connection $\nabla^{\perp}$. Assume that there exist some tensors $f, h, s, t$ and $B$ satisfying Equations (6)-(13) (note that (14) is not required since it is the dual equation of (12)) and the Gauss-Codazzi-Ricci equations (15)-(17). Moreover we define the operator $F: T M \oplus E \longrightarrow T M \oplus E$ by relations (5). If in addition the operator $F$ satisfy that the ranks of the maps $\frac{F+I d}{2}$ and $\frac{F-I d}{2}$ are 2 and 2 (resp. 3 and 1 ), then Kowalczyk [5] and De Lira-Tojeiro-Vitório [8] proved independently that there exists an isometric immersion from $(M, g)$ into $\mathbb{M}^{2}(c) \times \mathbb{R}^{2}$ (resp. $\left.\mathbb{M}^{3}(c) \times \mathbb{R}\right)$ with $E$ as normal bundle, $B$ as second fundamental form and such that the product structure of $\mathbb{M}^{2}(c) \times \mathbb{R}^{2}$ (resp. $\mathbb{M}^{3}(c) \times \mathbb{R}$ ) coincide with $F$ over $M$. Note that this was previously proven in a more abstract way by Piccione and Tausk [11].

2.2. Spinors on surfaces of $P$. For details about the recalls of this section, the reader can refer to [3] for instance. Let $\left(M^{2}, g\right)$ be an oriented Riemannian surface, with a given spin structure, and $E$ an oriented and spin vector bundle of rank 2 on $M$. We consider the spinor bundle $\Sigma$ over $M$ twisted by $E$ and defined by

$$
\Sigma=\Sigma M \otimes \Sigma E,
$$

where $\Sigma M$ and $\Sigma E$ are the spinor bundles of $M$ and $E$ respectively. We endow $\Sigma$ with the spinorial connection $\nabla$ defined by

$$
\nabla=\nabla^{\Sigma M} \otimes I d_{\Sigma E}+I d_{\Sigma M} \otimes \nabla^{\Sigma E} .
$$

We also define the Clifford product $\cdot$ by

$$
\begin{cases}X \cdot \varphi=\left(X \cdot{ }_{M} \alpha\right) \otimes \bar{\sigma} & \text { if } X \in \Gamma(T M) \\ X \cdot \varphi=\alpha \otimes\left(X{ }_{E} \sigma\right) & \text { if } X \in \Gamma(E)\end{cases}
$$

for all $\varphi=\alpha \otimes \sigma \in \Sigma M \otimes \Sigma E$, where $\cdot_{M}$ and $\cdot_{E}$ denote the Clifford products on $\Sigma M$ and on $\Sigma E$ respectively and where $\bar{\sigma}=\sigma^{+}-\sigma^{-}$for the natural decomposition of $\Sigma E=$ $\Sigma^{+} E \oplus \Sigma^{-} E$. Here, $\Sigma^{+} E$ and $\Sigma^{-} E$ are the eigensubbundles (for the eigenvalue 1 and -1 ) of $\Sigma E$ for the action of the normal volume element $\omega_{\perp}=i \xi_{1} \cdot{ }_{E} \xi_{2}$, where $\left\{\xi_{1}, \xi_{2}\right\}$ is a local orthonormal frame of $E$. Note that $\Sigma^{+} M$ and $\Sigma^{-}$are defined similarly by for the tangent volume element $\omega=i e_{1} \cdot{ }_{M} e_{2}$. We finally define the Dirac operator $D$ on $\Gamma(\Sigma)$ by

$$
D \varphi=e_{1} \cdot \nabla_{e_{1}} \varphi+e_{2} \cdot \nabla_{e_{2}} \varphi
$$

where $\left\{e_{1}, e_{2}\right\}$ is an orthonormal basis of $T M$.

We note that $\Sigma$ is also naturally equipped with a hermitian scalar product $\langle.,$.$\rangle which is$ compatible to the connection $\nabla$, since so are $\Sigma M$ and $\Sigma E$, and thus also with a compatible real scalar product $\mathfrak{R} e\langle.,$.$\rangle . We also note that the Clifford product - of vectors belonging$ to $T M \oplus E$ is antihermitian with respect to this hermitian product. Finally, we stress that the four subbundles $\Sigma^{ \pm \pm}:=\Sigma^{ \pm} M \otimes \Sigma^{ \pm} E$ are orthogonal with respect to the hermitian product. We will also consider $\Sigma^{+}=\Sigma^{++} \oplus \Sigma^{--}$and $\Sigma^{-}=\Sigma^{+-} \oplus \Sigma^{-+}$. Throughout the paper we will assume that the hermitian product is $\mathbb{C}$-linear w.r.t. the first entry, and $\mathbb{C}$-antilinear w.r.t. the second entry.

Now, let $(P, \widetilde{g})$ be a 4-dimensional spin manifold. It is a well-known fact that there 
is an identification between the spinor bundle $\Sigma P_{\mid M}$ of $P$ over $M$, and the spinor bundle of $M$ twisted by the normal bundle $\Sigma:=\Sigma M \otimes \Sigma E$. Moreover, we have the spinorial Gauss formula: for any $\varphi \in \Gamma(\Sigma)$ and any $X \in T M$,

$$
\widetilde{\nabla}_{X} \varphi=\nabla_{X} \varphi+\frac{1}{2} \sum_{j=1,2} e_{j} \cdot B\left(X, e_{j}\right) \cdot \varphi
$$

where $\widetilde{\nabla}$ is the spinorial connection of $\Sigma P$ and $\nabla$ is the spinoral connection of $\Sigma$ defined as above and $\left\{e_{1}, e_{2}\right\}$ is a local orthonormal frame of $T M$. We will also use this notation and $\left\{\xi_{1}, \xi_{2}\right\}$ for a local orthonormal frame of $E$. Here $\cdot$ is the Clifford product on $P$.

From now on, we will take $P=\mathbb{M}^{2}(c) \times \mathbb{R}^{2}$ or $\mathbb{M}^{3}(c) \times \mathbb{R}$. By restriction of a parallel spinor of the Euclidean space $\mathbb{R}^{5}$ if $c>0$ or the Lorentzian space $\mathbb{R}^{4,1}$ if $c<0$, we obtain on $P$ a spinor field $\varphi$ satisfying

$$
\left\{\begin{array}{lll}
\widetilde{\nabla}_{X} \varphi=\alpha X \cdot \varphi & \text { if } \quad X \in \Gamma\left(T \mathbb{M}^{2}(c)\right) \quad \text { or } \quad \Gamma\left(T \mathbb{M}^{3}(c)\right), \\
\widetilde{\nabla}_{X} \varphi=0 & \text { if } \quad X \in \Gamma\left(T \mathbb{R}^{2}\right) \text { or } \quad \Gamma(T \mathbb{R}(c)),
\end{array}\right.
$$

with $\alpha \in \mathbb{C}$ so that $4 \alpha^{2}=c$. In other words, for any $X \in \Gamma(T P)$, we have

$$
\widetilde{\nabla}_{X} \varphi=\frac{\alpha}{2}(X+F X) \cdot \varphi \text {. }
$$

Hence, by the spinorial Gauss formula (18), the restriction of $\varphi$ on $M$ satisfies

$$
\nabla_{X} \varphi=\frac{\alpha}{2}(X+f X+h X) \cdot \varphi+\eta(X) \cdot \varphi
$$

where $\eta(X)=-\frac{1}{2} \sum_{j=1}^{2} e_{j} \cdot B\left(e_{j}, X\right)$.

\section{MAIN RESUlt}

Now, we have the ingredients to state the the main result of this note.

Theorem 3.1. Let $c \in \mathbb{R}, c \neq 0$ and $\alpha \in \mathbb{C}$ such that $4 \alpha^{2}=c$. Let $\left(M^{2}, g\right)$ be an oriented Riemannian surface and $E$ an oriented vector bundle of rank 2 over $M$ with scalar product $<\cdot, \cdot\rangle_{E}$ and compatible connection $\nabla^{E}$. We denote by $\Sigma=\Sigma M \otimes \Sigma E$ the twisted spinor bundle. Let $B: T M \times T M \longrightarrow E$ a bilinear symmetric map and

$$
f: T M \longrightarrow T M, h: T M \longrightarrow E, s: E \longrightarrow T M \text { and } t: E \longrightarrow E
$$

satisfying Equations (6)-(13). Moreover we assume that the rank of the maps $\frac{F+I d}{2}$ and $\frac{F-I d}{2}$ is 2 and 2 (resp. 3 and 1), where $F: T M \oplus E \longrightarrow T M \oplus E$ is defined from $f, h, s$ and $t$ by relations (5). Then, the two following statements are equivalent

(1) There exists an isometric immersion of $\left(M^{2}, g\right)$ into $P=\mathbb{M}^{2}(c) \times \mathbb{R}^{2}$ (resp. $\left.\mathbb{M}^{3}(c) \times \mathbb{R}\right)$ with $E$ as normal bundle and second fundamental form $B$ such that over $M$ the product strcuture is given by $f, h, t$ and $s$.

(2) There exists a spinor field $\varphi$ in $\Sigma$ satisfying for all $X \in \mathfrak{X}(M)$

$$
\nabla_{X} \varphi=\frac{\alpha}{2}(X+f X+h X) \cdot \varphi+\eta(X) \cdot \varphi,
$$

such that $\varphi^{+}$and $\varphi^{-}$never vanish.

Proof: First, we remark that the fact that (1) implies (2) has been proved in the discussion of Section 2. The work consists in proving that (2) implies (1). The computations are in the same spirit as in [2], with some techinical difficulties due to the terms arising from the product structure. We will emphasize on these differences. We have to compute the spinorial curvature of the particular spinor $\varphi$. For this, let us compute $\mathcal{R}\left(e_{1}, e_{2}\right) \varphi$, where 
$\left(e_{1}, e_{2}\right)$ is a local orthonormal frame of $T M$. We also denote by $\left(e_{3}, e_{4}\right)$ a local orthonormal frame of $E$. Then, we have

$$
\begin{aligned}
\mathcal{R}\left(e_{1}, e_{2}\right) \varphi= & d^{\nabla} \eta\left(e_{1}, e_{2}\right) \cdot \varphi+\left(\eta\left(e_{2}\right) \cdot \eta\left(e_{1}\right)-\eta\left(e_{1}\right) \eta\left(e_{2}\right)\right) \cdot \varphi \\
& -\frac{\alpha}{2}\left(\nabla_{e_{2}} e_{1}+\nabla_{e_{2}}\left(f e_{1}\right)+\nabla_{e_{2}}^{\perp}\left(h e_{1}\right)\right) \cdot \varphi \\
& +\frac{\alpha}{2}\left(\nabla_{e_{1}} e_{2}+\nabla_{e_{1}}\left(f e_{2}\right)+\nabla_{e_{1}}^{\perp}\left(h e_{2}\right)\right) \cdot \varphi \\
& +\frac{\alpha^{2}}{4}\left(e_{2}+f e_{2}+h e_{2}\right) \cdot\left(e_{1}+f e_{1}+h e_{1}\right) \cdot \varphi \\
& -\frac{\alpha^{2}}{4}\left(e_{1}+f e_{1}+h e_{1}\right) \cdot\left(e_{2}+f e_{2}+h e_{2}\right) \cdot \varphi, \\
& +\frac{\alpha}{2}\left(\eta\left(e_{1}\right) \cdot\left(e_{2}+f e_{2}+h e_{2}\right)-\left(e_{2}+f e_{2}+h e_{2}\right) \cdot \eta\left(e_{1}\right)\right) \cdot \varphi \\
& -\frac{\alpha}{2}\left(\eta\left(e_{2}\right) \cdot\left(e_{1}+f e_{1}+h e_{1}\right)-\left(e_{1}+f e_{1}+h e_{1}\right) \cdot \eta\left(e_{2}\right)\right) \cdot \varphi \\
& -\frac{\alpha}{2}\left(\left[e_{1}, e_{2}\right]+f\left[e_{1}, e_{2}\right]+h\left[e_{1}, e_{2}\right]\right) \cdot \varphi
\end{aligned}
$$

where we denote $d^{\nabla} \eta(X, Y)=\nabla_{X}(\eta(Y))-\nabla_{Y}(\eta(X))-\eta([X, Y])$. First, by a straightforward computation, we see that the term

$$
\begin{aligned}
& +\frac{\alpha}{2}\left(\eta\left(e_{1}\right) \cdot\left(e_{2}+f e_{2}+h e_{2}\right)-\left(e_{2}+f e_{2}+h e_{2}\right) \cdot \eta\left(e_{1}\right)\right) \cdot \varphi \\
& -\frac{\alpha}{2}\left(\eta\left(e_{2}\right) \cdot\left(e_{1}+f e_{1}+h e_{1}\right)-\left(e_{1}+f e_{1}+h e_{1}\right) \cdot \eta\left(e_{2}\right)\right) \cdot \varphi
\end{aligned}
$$

vanishes. Moreover, by Equations (11) and (12) and the fact that the Levi-civita is torsionfree, the term

$$
\begin{aligned}
& \frac{\alpha}{2}\left(\nabla_{e_{1}} e_{2}+\nabla_{e_{1}}\left(f e_{2}\right)+\nabla_{e_{1}}^{\perp}\left(h e_{2}\right)\right) \cdot \varphi-\frac{\alpha}{2}\left(\nabla_{e_{2}} e_{1}+\nabla_{e_{2}}\left(f e_{1}\right)+\nabla_{e_{2}}^{\perp}\left(h e_{1}\right)\right) \cdot \varphi \\
& -\frac{\alpha}{2}\left(\left[e_{1}, e_{2}\right]+f\left[e_{1}, e_{2}\right]+h\left[e_{1}, e_{2}\right]\right) \cdot \varphi
\end{aligned}
$$

also vanishes. Hence, we get

$$
\begin{aligned}
\mathcal{R}\left(e_{1}, e_{2}\right) \varphi= & d^{\nabla} \eta\left(e_{1}, e_{2}\right) \cdot \varphi+\left(\eta\left(e_{2}\right) \cdot\left(e_{1}\right)-\eta\left(e_{1}\right) \eta\left(e_{2}\right)\right) \cdot \varphi \\
& +\frac{\alpha^{2}}{2}\left(\left\langle f e_{1}, e_{2}\right\rangle^{2}-\left\langle f e_{1}, e_{1}\right\rangle\left\langle f e_{2}, e_{2}\right\rangle\right) e_{1} \cdot e_{2} \cdot \varphi \\
& +\frac{\alpha^{2}}{2}\left(\left\langle h e_{2}, e_{3}\right\rangle\left\langle f e_{1}, e_{4}\right\rangle-\left\langle h e_{1}, e_{3}\right\rangle\left\langle h e_{2}, e_{4}\right\rangle\right) e_{3} \cdot e_{4} \cdot \varphi \\
& +\frac{\alpha^{2}}{2}\left(f e_{2} \cdot h e_{1}-f e_{1} \cdot h e_{2}-e_{2} \cdot h e_{1}+e_{1} \cdot h e_{2}\right) \cdot \varphi
\end{aligned}
$$

But, as computed in [2], we have

$$
\begin{gathered}
\mathcal{R}\left(e_{1}, e_{2}\right) \varphi=-\frac{1}{2} K e_{1} \cdot e_{2} \cdot \varphi-\frac{1}{2} K_{N} e_{3} \cdot e_{4} \cdot \varphi, \\
d^{\nabla} \eta(X, Y)=-\frac{1}{2} \sum_{j=1}^{2} e_{j} \cdot\left(\left(\bar{\nabla}_{X} B\right)\left(Y, e_{j}\right)-\left(\bar{\nabla}_{Y} B\right)\left(X, e_{j}\right)\right),
\end{gathered}
$$

where $\bar{\nabla}$ stands for the natural connection on $T^{*} M \otimes T^{*} M \otimes E$, and

$$
\begin{aligned}
\eta\left(e_{2}\right) \cdot \eta\left(e_{1}\right)-\eta\left(e_{1}\right) \cdot \eta\left(e_{2}\right)= & \frac{1}{2}\left(\left|B\left(e_{1}, e_{2}\right)\right|^{2}-\left\langle B\left(e_{1}, e_{1}\right), B\left(e_{2}, e_{2}\right)\right\rangle\right) e_{1} \cdot e_{2} \\
& +\frac{1}{2}\left\langle\left(S_{e_{3}} \circ S_{e_{4}}-S_{e_{4}} \circ S_{e_{3}}\right)\left(e_{1}\right), e_{2}\right\rangle e_{3} \cdot e_{4} .
\end{aligned}
$$

Therefore, we have

$$
G \cdot \varphi+R \cdot \varphi+C \cdot \varphi=0
$$


where $G, R$ and $C$ are the 2-forms defined by

$$
\begin{aligned}
G= & {\left[K+\left\langle B\left(e_{1}, e_{1}\right), B\left(e_{2}, e_{2}\right)\right\rangle-\left|B\left(e_{1}, e_{2}\right)\right|\right.} \\
& \left.+\alpha^{2}\left(1-\left\langle f e_{1}, e_{2}\right\rangle^{2}+\left\langle f e_{1}, e_{1}\right\rangle\left\langle f e_{2}, e_{2}\right\rangle\right)\right] e_{1} \cdot e_{2},
\end{aligned}
$$

where $K$ is the Gauss curvature of $(M, g)$,

$$
\begin{aligned}
R= & {\left[K_{E}+\left\langle\left(S_{e_{3}} \circ S_{e_{4}}-S_{e_{4}} \circ S_{e_{3}}\right)\left(e_{1}\right), e_{2}\right\rangle\right.} \\
& \left.+\alpha^{2}\left(\left\langle h e_{1}, e_{3}\right\rangle\left\langle h e_{2}, e_{4}\right\rangle-\left\langle h e_{1}, e_{4}\right\rangle\left\langle f e_{2}, e_{3}\right\rangle\right)\right] e_{3} \cdot e_{4},
\end{aligned}
$$

where $K_{E}$ is the curvature of the bundle $E$, and

$$
C=2 d^{\nabla} \eta\left(e_{1}, e_{2}\right)+\alpha^{2}\left(f e_{2} \cdot h e_{1}-f e_{1} \cdot h e_{2}+e_{2} \cdot h e_{1}-e_{1} \cdot h e_{2}\right) .
$$

As proved in [2], if $T$ is a 2-form such that $T \cdot \varphi=0$ with $\varphi^{+}$and $\varphi^{-}$nowhere vanishing, then $T=0$. Moreover, since $G$ belongs to $\Lambda^{2} M \otimes 1, R$ belongs to $1 \otimes \Lambda^{2} E$ and $C$ is of mixed type, that is, belongs to $T M \otimes E$, then each of these three parts are zero. But $G=0$ is nothing else but

$$
K+\left\langle B\left(e_{1}, e_{1}\right), B\left(e_{2}, e_{2}\right)\right\rangle-\left|B\left(e_{1}, e_{2}\right)\right|=-\frac{c}{4}\left(1-\left\langle f e_{1}, e_{2}\right\rangle^{2}+\left\langle f e_{1}, e_{1}\right\rangle\left\langle f e_{2}, e_{2}\right\rangle\right),
$$

that is the Gauss equation. Similarly, $R=0$ is equivalent to

$$
K_{E}+\left\langle\left(S_{e_{3}} \circ S_{e_{4}}-S_{e_{4}} \circ S_{e_{3}}\right)\left(e_{1}\right), e_{2}\right\rangle=-\frac{c}{4}\left(\left\langle h e_{1}, e_{3}\right\rangle\left\langle h e_{2}, e_{4}\right\rangle-\left\langle h e_{1}, e_{4}\right\rangle\left\langle f e_{2}, e_{3}\right\rangle\right),
$$

That is the Ricci equation. Finally $C=0$, gives the Codazzi equations. Indeed, since

$$
d^{\nabla} \eta(X, Y)=-\frac{1}{2} \sum_{j=1}^{2} e_{j} \cdot\left(\left(\bar{\nabla}_{X} B\right)\left(Y, e_{j}\right)-\left(\bar{\nabla}_{Y} B\right)\left(X, e_{j}\right)\right) .
$$

Thus, from $C=0$, we deduce for $j=1,2$

$$
\begin{aligned}
\left(\bar{\nabla}_{e_{1}} B\right)\left(e_{2}, e_{j}\right)-\left(\bar{\nabla}_{e_{2}} B\right)\left(e_{1}, e_{j}\right)= & \frac{c}{4}\left[\left\langle f e_{2}, e_{j}\right\rangle h e_{1}-\left\langle f e_{1}, e_{j}\right\rangle h e_{2}\right. \\
& \left.+\left\langle e_{2}, e_{j}\right\rangle h e_{1}-\left\langle e_{1}, e_{j}\right\rangle h e_{2}\right],
\end{aligned}
$$

which are the Coazzi equations. Since in addition, we have assumed Equations (6)-(12), by the theorem of Kowalczyk and De Lira-Tojeiro-Vitório, we get that $\left(M^{2}, g\right)$ is isometrically immersed into $P$ with $B$ as second fundamental form and $f, h, s$ and $t$ coming from the product structure $F$ of $P$. This concludes the proof.

Remark 3.2. Note that in the proof, we only use Equations (11) and (12) in the computations. The other Equations (6)-(10) and (13)-(13) are only needed to apply the theorem of Kowalczyk and De Lira-Tojeiro-Vitori, as well as the hypothesis on the rank of the maps $\frac{F+I d}{2}$ and $\frac{F-I d}{2}$.

\section{THE DIRAC EQUATION}

Let $\varphi$ be a spinor field satisfying Equation (19), then it satisfies the following Dirac equation

$$
D \varphi=\vec{H} \cdot \varphi-\frac{\alpha}{2}[(2+\operatorname{tr}(f)) \varphi-\beta \cdot \varphi],
$$

where $\beta$ is the 2 -form defined by $\beta=\sum_{i=1,2} e_{i} \cdot h e_{i}=\sum_{i, j=1}^{2} h_{i j} e_{i} \cdot \xi_{j}$, where $h_{i, j}=\left\langle h e_{i}, \xi_{j}\right\rangle$. As in [2], we will show that this equation with an appropiate condition on the norm of both $\varphi^{+}$and $\varphi^{-}$is equivalent to Equation (19), where the tensor $B$ is expressed in terms on the 
spinor field $\varphi$ and such that $\operatorname{tr}(B)=2 \vec{H}$. Moreover, from Equation (19) we deduce the following conditions on the derivatives of $\left|\varphi^{+}\right|^{2}$ and $\left|\varphi^{-}\right|^{2}$. Indeed, after decomposition onto $\Sigma^{+}$and $\Sigma^{-}$, (19) becomes

$$
\nabla_{X} \varphi^{ \pm}=\frac{\alpha}{2}(X+f X+h X) \cdot \varphi^{\mp}+\eta(X) \cdot \varphi^{ \pm} .
$$

From this we deduce that

$$
X\left(\left|\varphi^{ \pm}\right|^{2}\right)=\mathfrak{R} e\left\langle\alpha(X+f X+h X) \cdot \varphi^{\mp}, \varphi^{ \pm}\right\rangle
$$

Now, let $\varphi$ a spinor field solution of the Dirac equation (24) with $\varphi^{+}$and $\varphi^{-}$nowhere vanishing and satisying the norm condition (25), we set for any vector fields $X$ and $Y$ tangent to $M$ and $\xi \in E$

$$
\begin{aligned}
\left\langle B^{+}(X, Y), \xi\right\rangle=\frac{1}{2\left|\varphi^{+}\right|^{2}}\left[\frac{\alpha}{2}\langle(X \cdot f Y\right. & \left.+Y \cdot f X) \cdot \varphi^{-}+(X \cdot h Y+Y \cdot h X) \cdot \varphi^{-}, \xi \cdot \varphi^{+}\right\rangle \\
& \left.+\left\langle X \cdot \nabla_{Y} \varphi^{+}+\alpha<X, Y>\varphi^{-}, \xi \cdot \varphi^{+}\right\rangle\right]
\end{aligned}
$$

and

(27)

$$
\begin{gathered}
\left\langle B^{-}(X, Y), \xi\right\rangle=\frac{1}{2\left|\varphi^{-}\right|^{2}}\left[\frac{\alpha}{2}\left\langle(X \cdot j Y+Y \cdot j X) \cdot \varphi^{+}+(X \cdot h Y+Y \cdot h X) \cdot \varphi^{+}, \xi \cdot \varphi^{-}\right\rangle\right. \\
\left.+\left\langle X \cdot \nabla_{Y} \varphi^{-}+\alpha<X, Y>\varphi^{+}, \xi \cdot \varphi^{-}\right\rangle\right] .
\end{gathered}
$$

Finally, we set $B=B^{+}+B^{-}$. Then, we have the following

Proposition 4.1. Let $\varphi \in \Gamma(\Sigma)$ satisfying the Dirac equation (24)

$$
D \varphi=\vec{H} \cdot \varphi-\frac{\alpha}{2}[(2+\operatorname{tr}(f)) \varphi-\beta \cdot \varphi]
$$

such that

$$
X\left(\left|\varphi^{ \pm}\right|^{2}\right)=\mathfrak{R} e\left\langle\alpha(X+f X+h X) \cdot \varphi^{\mp}, \varphi^{ \pm}\right\rangle
$$

then $\varphi$ is solution of Equation (19)

$$
\nabla_{X} \varphi=\frac{\alpha}{2}(X+f X+h X) \cdot \varphi+\eta(X) \cdot \varphi
$$

where $\eta$ is defined by $\eta(X)=-\frac{1}{2} \sum_{j=1}^{2} e_{j} \cdot B\left(e_{j}, X\right)$.

For the sake of clarity, the proof of this proposition will be given in the next section. Now, combining this proposition with Theorem 3.1, we get the following corollary.

Corollary 4.2. Let $c \in \mathbb{R}, c \neq 0$ and $\alpha \in \mathbb{C}$ such that $4 \alpha^{2}=c$. Let $\left(M^{2}, g\right)$ be an oriented Riemannian surface and $E$ an oriented vector bundle of rank 2 over $M$ with scalar product $\langle\cdot, \cdot\rangle_{E}$ and compatible connection $\nabla^{E}$. We denote by $\Sigma=\Sigma M \otimes \Sigma E$ the twisted spinor bundle. Let $f, h, s$ and s be some maps

$$
f: T M \longrightarrow T M, h: T M \longrightarrow E, s: E \longrightarrow T M \text { and } t: E \longrightarrow E
$$

satisfying Equations (6)-(10). Moreover we assume that the rank of the maps $\frac{F+I d}{2}$ and $\frac{F-I d}{2}$ are 2 and 2 (resp. 3 and 1), where $F: T M \oplus E \longrightarrow T M \oplus E$ is defined by relations (5). Then, the two following statements are equivalent 
(1) There exists an isometric immersion of $\left(M^{2}, g\right)$ into $\mathbb{M}^{2}(c) \times \mathbb{R}^{2}\left(\right.$ resp. $\left.\mathbb{M}^{3}(c) \times \mathbb{R}\right)$ with $E$ as normal bundle and mean curvature $\vec{H}$ such that over $M$ the product strcuture is given by $f, h, t$ and $s$.

(2) There exists a spinor field $\varphi$ in $\Sigma$ solution of the Dirac equation

$$
D \varphi=\vec{H} \cdot \varphi-\frac{\alpha}{2}[(2+\operatorname{tr}(f)) \varphi-\beta \cdot \varphi]
$$

such that $\varphi^{+}$and $\varphi^{-}$never vanish, satisfy the norm condition (25) and such that the maps $f, h, s, t$ and the tensor $B$ defined by (26) and (27) satisfy relations (11)(13).

\section{Proof of Proposition 4.1}

First, we decompose the Dirac equation (24) on the four spinor subbundles $\Sigma^{++}, \Sigma^{--}$, $\Sigma^{+-}$and $\Sigma^{-+}$. We get the following four equations

$$
\left\{\begin{array}{l}
D \varphi^{--}=\vec{H} \cdot \varphi^{++}-\frac{\alpha}{2}(2+\operatorname{tr}(f)) \varphi^{+-}+\frac{\alpha}{2} \beta \cdot \varphi^{-+} \\
D \varphi^{++}=\vec{H} \cdot \varphi^{--}-\frac{\alpha}{2}(2+\operatorname{tr}(f)) \varphi^{-+}+\frac{\alpha}{2} \beta \cdot \varphi^{+-} \\
D \varphi^{+-}=\vec{H} \cdot \varphi^{-+}-\frac{\alpha}{2}(2+\operatorname{tr}(f)) \varphi^{--}+\frac{\alpha}{2} \beta \cdot \varphi^{++} \\
D \varphi^{-+}=\vec{H} \cdot \varphi^{+-}-\frac{\alpha}{2}(2+\operatorname{tr}(f)) \varphi^{++}+\frac{\alpha}{2} \beta \cdot \varphi^{--}
\end{array}\right.
$$

Now, we fix a point $p \in M$, and consider $e_{3}$ a unit vector in $E_{p}$ so that the mean curvature vector is given by $\vec{H}=|\vec{H}| e_{3}$ at $p$. We complete $e_{3}$ by $e_{4}$ to get a positively oriented and orthonormal frame of $E_{p}$. First, we assume that $\varphi^{--}, \varphi^{++}, \varphi^{+-}$and $\varphi^{-+}$do not vanish at $p$. It is easy to see that

$$
\left\{e_{1} \cdot e_{3} \cdot \frac{\varphi^{--}}{\left|\varphi^{--}\right|}, e_{2} \cdot e_{3} \cdot \frac{\varphi^{--}}{\left|\varphi^{--}\right|}\right\}
$$

is an orthonormal frame of $\Sigma^{++}$for the real scalar product $\mathfrak{R} e\langle\cdot, \cdot\rangle$. Indeed, we have

$$
\begin{aligned}
\mathfrak{R} e\left\langle e_{1} \cdot e_{3} \cdot \varphi^{--}, e_{2} \cdot e_{3} \cdot \varphi^{--}\right\rangle & =\mathfrak{R} e\left\langle\varphi^{--}, e_{3} \cdot e_{1} \cdot e_{2} \cdot e_{3} \cdot \varphi^{--}\right\rangle \\
& =\mathfrak{R} e\left(i\left|\varphi^{--}\right|^{2}\right)=0 .
\end{aligned}
$$

Of course, by the same argument,

$$
\begin{aligned}
& \left\{e_{1} \cdot e_{3} \cdot \frac{\varphi^{++}}{\left|\varphi^{++}\right|}, e_{2} \cdot e_{3} \cdot \frac{\varphi^{++}}{\left|\varphi^{++}\right|}\right\}, \\
& \left\{e_{1} \cdot e_{3} \cdot \frac{\varphi^{-+}}{\left|\varphi^{-+}\right|}, e_{2} \cdot e_{3} \cdot \frac{\varphi^{-+}}{\left|\varphi^{-+}\right|}\right\}, \\
& \left\{e_{1} \cdot e_{3} \cdot \frac{\varphi^{+-}}{\left|\varphi^{+-}\right|}, e_{2} \cdot e_{3} \cdot \frac{\varphi^{+-}}{\left|\varphi^{+-}\right|}\right\}
\end{aligned}
$$

are orthonormal frames of $\Sigma^{--}, \Sigma^{+-}$and $\Sigma^{-+}$respectively. We define the following bilinear forms

$$
\begin{aligned}
& F_{++}(X, Y)=\mathfrak{R} e\left\langle\nabla_{X} \varphi^{++}, Y \cdot e_{3} \cdot \varphi^{--}\right\rangle, \\
& F_{--}(X, Y)=\mathfrak{R} e\left\langle\nabla_{X} \varphi^{--}, Y \cdot e_{3} \cdot \varphi^{++}\right\rangle, \\
& F_{+-}(X, Y)=\mathfrak{R} e\left\langle\nabla_{X} \varphi^{+-}, Y \cdot e_{3} \cdot \varphi^{-+}\right\rangle, \\
& F_{-+}(X, Y)=\mathfrak{R} e\left\langle\nabla_{X} \varphi^{-+}, Y \cdot e_{3} \cdot \varphi^{+-}\right\rangle,
\end{aligned}
$$

and

$$
\begin{aligned}
& B_{++}(X, Y)=-\frac{1}{2} \mathfrak{R} e\left\langle\alpha(X+f X) \cdot \varphi^{-+}+\alpha h X \cdot \varphi^{+-}, Y \cdot e_{3} \cdot \varphi^{--}\right\rangle, \\
& B_{--}(X, Y)=-\frac{1}{2} \mathfrak{R} e\left\langle\alpha(X+f X) \cdot \varphi^{+-}+\alpha h X \cdot \varphi^{-+}, Y \cdot e_{3} \cdot \varphi^{++}\right\rangle, \\
& B_{+-}(X, Y)=-\frac{1}{2} \mathfrak{R} e\left\langle\alpha(X+f X) \cdot \varphi^{++}+\alpha h X \cdot \varphi^{--}, Y \cdot e_{3} \cdot \varphi^{+-}\right\rangle,
\end{aligned}
$$




$$
B_{-+}(X, Y)=-\frac{1}{2} \mathfrak{R} e\left\langle\alpha(X+f X) \cdot \varphi^{--}+\alpha h X \cdot \varphi^{++}, Y \cdot e_{3} \cdot \varphi^{-+}\right\rangle .
$$

We have this first lemma:

Lemma 5.1. We have

(1) $\operatorname{tr}\left(F_{++}\right)=-|\vec{H}|\left|\varphi^{--}\right|^{2}+\frac{1}{2} \mathfrak{R} e\left\langle\alpha(2+\operatorname{tr}(f)) \varphi^{-+}+\alpha \beta \cdot \varphi^{+-}, e_{3} \cdot \varphi^{--}\right\rangle$,

(2) $\operatorname{tr}\left(F_{--}\right)=-|\vec{H}|\left|\varphi^{++}\right|^{2}+\frac{1}{2} \mathfrak{R} e\left\langle\alpha(2+\operatorname{tr}(f)) \varphi^{+-}+\alpha \beta \cdot \varphi^{-+}, e_{3} \cdot \varphi^{++}\right\rangle$,

(3) $\operatorname{tr}\left(F_{+-}\right)=-|\vec{H}|\left|\varphi^{-+}\right|^{2}+\frac{1}{2} \mathfrak{R} e\left\langle\alpha(2+\operatorname{tr}(f)) \varphi^{++}+\alpha \beta \cdot \varphi^{--}, e_{3} \cdot \varphi^{-+}\right\rangle$,

(4) $\operatorname{tr}\left(F_{-+}\right)=-\left|\vec{H} \| \varphi^{+-}\right|^{2}+\frac{1}{2} \mathfrak{R} e\left\langle\alpha(2+\operatorname{tr}(f)) \varphi^{--}+\alpha \beta \cdot \varphi^{++}, e_{3} \cdot \varphi^{+-}\right\rangle$,

Proof: We only compute the trace of $F_{++}$, the computations for the three others forms $F_{--}, F_{+-}$and $F_{-+}$are the same. We have

$$
\begin{aligned}
\operatorname{tr}\left(F_{++}\right) & =F_{++}\left(e_{1}, e_{1}\right)+F_{++}\left(e_{2}, e_{2}\right) \\
& =\mathfrak{R} e\left\langle\nabla_{e_{1}} \varphi^{++}, e_{1} \cdot e_{3} \cdot \varphi^{--}\right\rangle+\mathfrak{R} e\left\langle\nabla_{e_{2}} \varphi^{++}, e_{2} \cdot e_{3} \cdot \varphi^{--}\right\rangle \\
& =-\mathfrak{R} e\left\langle e_{1} \cdot \nabla_{e_{1}} \varphi^{++}, e_{3} \cdot \varphi^{--}\right\rangle-\mathfrak{R} e\left\langle e_{2} \cdot \nabla_{e_{2}} \varphi^{++}, e_{3} \cdot \varphi^{--}\right\rangle \\
& =-\mathfrak{R} e\left\langle D \varphi^{++}, e_{3} \cdot \varphi^{--}\right\rangle
\end{aligned}
$$

Since $D \varphi^{++}=\vec{H} \cdot \varphi^{--}-\frac{\alpha}{2}(2+\operatorname{tr}(f)) \varphi^{-+}+\frac{\alpha}{2} \beta \cdot \varphi^{+-}$, we get

$$
\begin{aligned}
\operatorname{tr}\left(F_{++}\right) & =-\mathfrak{R} e\left\langle\vec{H} \cdot \varphi^{--}-\frac{\alpha}{2}(2+\operatorname{tr}(f)) \varphi^{-+}+\frac{\alpha}{2} \beta \cdot \varphi^{+-}, e_{3} \cdot \varphi^{--}\right\rangle \\
& =-\mathfrak{R} e\left\langle|H| e_{3} \cdot \varphi^{--}, e_{3} \cdot \varphi^{--}\right\rangle+\mathfrak{R} e\left\langle\frac{\alpha}{2}(2+\operatorname{tr}(f)) \varphi^{-+}-\frac{\alpha}{2} \beta \cdot \varphi^{+-}, e_{3} \cdot \varphi^{--}\right\rangle \\
& =-|\vec{H}|\left|\varphi^{--}\right|^{2}+\frac{1}{2} \mathfrak{R} e\left\langle\alpha(2+\operatorname{tr}(f)) \varphi^{-+}+\alpha \beta \cdot \varphi^{+-}, e_{3} \cdot \varphi^{--}\right\rangle
\end{aligned}
$$

This concludes the proof.

TNow, we have this second lemma which gives the defect of symmetry:

Lemma 5.2. We have

(1) $F_{++}\left(e_{1}, e_{2}\right)=F_{++}\left(e_{2}, e_{1}\right)-\frac{1}{2} \mathfrak{R} e\left\langle(2+\operatorname{tr}(f)) \varphi^{-+}-\alpha \beta \cdot \varphi^{+-}, e_{4} \cdot \varphi^{--}\right\rangle$,

(2) $F_{--}\left(e_{1}, e_{2}\right)=F_{--}\left(e_{2}, e_{1}\right)-\frac{1}{2} \mathfrak{R} e\left\langle(2+\operatorname{tr}(f)) \varphi^{+-}-\alpha \beta \cdot \varphi^{-+}, e_{4} \cdot \varphi^{++}\right\rangle$,

(3) $F_{+-}\left(e_{1}, e_{2}\right)=F_{+-}\left(e_{2}, e_{1}\right)+\frac{1}{2} \mathfrak{R} e\left\langle(2+\operatorname{tr}(f)) \varphi^{++}-\alpha \beta \cdot \varphi^{--}, e_{4} \cdot \varphi^{+-}\right\rangle$,

(4) $F_{-+}\left(e_{1}, e_{2}\right)=F_{-+}\left(e_{2}, e_{1}\right)+\frac{1}{2} \mathfrak{R} e\left\langle(2+\operatorname{tr}(f)) \varphi^{--}-\alpha \beta \cdot \varphi^{++}, e_{4} \cdot \varphi^{-+}\right\rangle$.

Proof: As for the proof of the previous lemma, we only give the details for $F_{++}$. We have

$$
\begin{aligned}
F_{++}\left(e_{1}, e_{2}\right) & =\mathfrak{R} e\left\langle\nabla_{e_{1}} \varphi^{++}, e_{2} \cdot e_{3} \cdot \varphi^{--}\right\rangle \\
& =\mathfrak{R} e\left\langle e_{1} \cdot \nabla_{e_{1}} \varphi^{++}, e_{1} \cdot e_{2} \cdot e_{3} \cdot \varphi^{--}\right\rangle \\
& =\mathfrak{R} e\left\langle\vec{H} \cdot \varphi^{--}-\frac{\alpha}{2}(2+\operatorname{tr}(f)) \varphi^{-+}+\frac{\alpha}{2} \beta \cdot \varphi^{+-}-e_{2} \cdot \nabla_{e_{2}} \varphi^{++}, e_{1} \cdot e_{2} \cdot e_{3} \cdot \varphi^{--}\right\rangle .
\end{aligned}
$$

The first term is

$$
\begin{aligned}
\mathfrak{R} e\left\langle\vec{H} \cdot \varphi^{--}, e_{1} \cdot e_{2} \cdot e_{3} \cdot \varphi^{--}\right\rangle & =-\mathfrak{R} e\left\langle e_{3} \cdot \vec{H} \cdot \varphi^{--}, e_{1} \cdot e_{2} \cdot \varphi^{--}\right\rangle \\
& =\mathfrak{R} e\left\langle\vec{H} \cdot e_{3} \cdot \varphi^{--}, i \varphi^{--}\right\rangle \\
& =-\mathfrak{R} e\left(i\left|\vec{H} \| \varphi^{--}\right|^{2}\right)=0,
\end{aligned}
$$


where we have use that $i e_{1} \cdot e_{2} \cdot \varphi^{--}=-\varphi^{--}$, that is, $e_{1} \cdot e_{2} \cdot \varphi^{--}=i \varphi^{--}$and $\vec{H}=|H| e_{3}$. Moreover, we have

$$
\begin{aligned}
-\mathfrak{R} e\left\langle e_{2} \cdot \nabla_{e_{2}} \varphi^{++}, e_{1} \cdot e_{2} \cdot e_{3} \cdot \varphi^{--}\right\rangle & =\mathfrak{R} e\left\langle\nabla_{e_{2}} \varphi^{++}, e_{2} \cdot e_{1} \cdot e_{2} \cdot e_{3} \cdot \varphi^{--}\right\rangle \\
& =\mathfrak{R} e\left\langle\nabla_{e_{2}} \varphi^{++}, e_{1} \cdot e_{3} \cdot \varphi^{--}\right\rangle \\
& =F_{++}\left(e_{2}, e_{1}\right) .
\end{aligned}
$$

Finally, since $\varphi^{--} \in \Sigma^{+}$, we have $\omega_{4} \cdot \varphi^{--}=\varphi^{--}$, which implies $e_{1} \cdot e_{2} \cdot e_{3} \cdot \varphi^{--}=$ $-e_{4} \cdot \varphi^{--}$and we get

$$
F_{++}\left(e_{1}, e_{2}\right)=F_{++}\left(e_{2}, e_{1}\right)-\frac{1}{2} \Re\left(\alpha(2+\operatorname{tr}(f)) \varphi^{-+}-\alpha \beta \cdot \varphi^{+-}, e_{4} \cdot \varphi^{--}\right\rangle .
$$

The proof is similar for the three other forms.

By analogous computations, we also get the following lemmas. We do not give the proof which is similar to the two previous ones.

Lemma 5.3. We have

(1) $\operatorname{tr}\left(B_{++}\right)=-\frac{1}{2} \mathfrak{R} e\left\langle\alpha(2+\operatorname{tr}(f)) \varphi^{-+}+\alpha \beta \cdot \varphi^{+-}, e_{3} \cdot \varphi^{--}\right\rangle$,

(2) $\operatorname{tr}\left(B_{--}\right)=-\frac{1}{2} \mathfrak{R} e\left\langle\alpha(2+\operatorname{tr}(f)) \varphi^{+-}+\alpha \beta \cdot \varphi^{-+}, e_{3} \cdot \varphi^{++}\right\rangle$,

(3) $\operatorname{tr}\left(B_{+-}\right)=-\frac{1}{2} \mathfrak{R} e\left\langle\alpha(2+\operatorname{tr}(f)) \varphi^{++}+\alpha \beta \cdot \varphi^{--}, e_{3} \cdot \varphi^{-+}\right\rangle$,

(4) $\operatorname{tr}\left(B_{-+}\right)=-\frac{1}{2} \mathfrak{R} e\left\langle\alpha(2+\operatorname{tr}(f)) \varphi^{--}+\alpha \beta \cdot \varphi^{++}, e_{3} \cdot \varphi^{+-}\right\rangle$.

Lemma 5.4. We have

(1) $B_{++}\left(e_{1}, e_{2}\right)=B_{++}\left(e_{2}, e_{1}\right)+\frac{1}{2} \mathfrak{R} e\left\langle(2+\operatorname{tr}(f)) \varphi^{-+}-\alpha \beta \cdot \varphi^{+-}, e_{4} \cdot \varphi^{--}\right\rangle$,

(2) $B_{--}\left(e_{1}, e_{2}\right)=B_{--}\left(e_{2}, e_{1}\right)+\frac{1}{2} \mathfrak{R} e\left\langle(2+\operatorname{tr}(f)) \varphi^{+-}-\alpha \beta \cdot \varphi^{-+}, e_{4} \cdot \varphi^{++}\right\rangle$,

(3) $B_{+-}\left(e_{1}, e_{2}\right)=B_{+-}\left(e_{2}, e_{1}\right)-\frac{1}{2} \mathfrak{R} e\left\langle(2+\operatorname{tr}(f)) \varphi^{++}-\alpha \beta \cdot \varphi^{--}, e_{4} \cdot \varphi^{+-}\right\rangle$,

(4) $B_{-+}\left(e_{1}, e_{2}\right)=B_{-+}\left(e_{2}, e_{1}\right)-\frac{1}{2} \mathfrak{R} e\left\langle(2+\operatorname{tr}(f)) \varphi^{--}-\alpha \beta \cdot \varphi^{++}, e_{4} \cdot \varphi^{-+}\right\rangle$.

Now, we set

$$
\left\{\begin{array}{l}
A_{++}:=F_{++}+B_{++}, \\
A_{--}:=F_{--}+B_{--}, \\
A_{+-}:=F_{+-}+B_{+-}, \\
A_{-+}:=F_{-+}+B_{-+},
\end{array}\right.
$$

and

$$
F_{+}=\frac{A_{++}}{\left|\varphi^{--}\right|^{2}}-\frac{A_{--}}{\left|\varphi^{++}\right|^{2}} \quad \text { and } \quad F_{-}=\frac{A_{+-}}{\left|\varphi^{-+}\right|^{2}}-\frac{A_{-+}}{\left|\varphi^{+-}\right|^{2}} .
$$

From the last four lemmas we deduce immediately that $F_{+}$and $F_{-}$are symmetric and trace-free. Moreover, by a direct computation using the conditions (25) on the norms of $\varphi^{+}$ and $\varphi^{-}$, we get the following lemma:

Lemma 5.5. The symmetric operators $F^{+}$and $F^{-}$of TM associated to the bilinear forms $F_{+}$and $F_{-}$, defined by

$F^{+}(X)=F_{+}\left(X, e_{1}\right) e_{1}+F_{+}\left(X, e_{2}\right) e_{2} \quad$ and $\quad F^{-}(X)=F_{-}\left(X, e_{1}\right) e_{1}+F_{-}\left(X, e_{2}\right) e_{2}$ for all $X \in T M$, satisfy

(1) $\mathfrak{R} e\left\langle F^{+}(X) \cdot e_{3} \cdot \varphi^{--}, \varphi^{++}\right\rangle=0$,

(2) $\mathfrak{R} e\left\langle F^{-}(X) \cdot e_{3} \cdot \varphi^{-+}, \varphi^{+-}\right\rangle=0$. 
Proof. First, we have

$$
A_{++}(X, Y)=\mathfrak{R} e\left\langle\nabla_{X} \varphi^{++}-\alpha(X+f X) \cdot \varphi^{-+}+\alpha h X \cdot \varphi^{+-}, Y \cdot e_{3} \cdot \varphi^{--}\right\rangle,
$$

Since $\left(e_{1} \cdot e_{3} \cdot \frac{\varphi^{--}}{\left|\varphi^{--}\right|}, e_{2} \cdot e_{3} \cdot \frac{\varphi^{--}}{\left|\varphi^{--}\right|}\right)$is an orthonormal frame of $\Sigma^{++}$, we have

$$
\begin{aligned}
& \mathfrak{R} e\left\langle\nabla_{X} \varphi^{++}-\frac{\alpha}{2}(X+f X) \cdot \varphi^{-+}+\frac{\alpha}{2} h X \cdot \varphi^{+-}, \varphi^{++}\right\rangle \\
= & \frac{A_{++}}{\left|\varphi^{--}\right|^{2}}\left(X, e_{1}\right) \mathfrak{R} e\left\langle e_{1} \cdot e_{3} \cdot \varphi^{--}, \varphi^{++}\right\rangle+\frac{A_{++}}{\left|\varphi^{--}\right|^{2}}\left(X, e_{2}\right) \mathfrak{R} e\left\langle e_{2} \cdot e_{3} \cdot \varphi^{--}, \varphi^{++}\right\rangle .
\end{aligned}
$$

Similarly,

$$
\begin{aligned}
& \mathfrak{R} e\left\langle\nabla_{X} \varphi^{--}-\frac{\alpha}{2}(X+f X) \cdot \varphi^{+-}+\frac{\alpha}{2} h X \cdot \varphi^{-+}, \varphi^{--}\right\rangle \\
& =\frac{A_{--}}{\left|\varphi^{++}\right|^{2}}\left(X, e_{1}\right) \mathfrak{R} e\left\langle e_{1} \cdot e_{3} \cdot \varphi^{++}, \varphi^{--}\right\rangle+\frac{A_{--}}{\left|\varphi^{++}\right|^{2}}\left(X, e_{2}\right) \mathfrak{R} e\left\langle e_{2} \cdot e_{3} \cdot \varphi^{++}, \varphi^{--}\right\rangle \\
& =-\frac{A_{--}}{\left|\varphi^{++}\right|^{2}}\left(X, e_{1}\right) \mathfrak{R} e\left\langle e_{1} \cdot e_{3} \cdot \varphi^{--}, \varphi^{++}\right\rangle-\frac{A_{--}}{\left|\varphi^{++}\right|^{2}}\left(X, e_{2}\right) \mathfrak{R} e\left\langle e_{2} \cdot e_{3} \cdot \varphi^{--}, \varphi^{++}\right\rangle .
\end{aligned}
$$

Summing these two formulas imply that

$$
\mathfrak{R} e\left\langle F^{+}(X) \cdot e_{3} \cdot \varphi^{--}, \varphi^{++}\right\rangle=\mathfrak{R} e\left\langle\nabla_{X} \varphi^{+}-\frac{\alpha}{2}(X+f X) \cdot \varphi^{-}+\frac{\alpha}{2} h X \cdot \varphi^{-}, \varphi^{+}\right\rangle .
$$

By the condition (25) on the derivative of the norm of $\varphi^{+}$, this last expression is zero. The proof of the second relation is similar.

Hence, the operators $F^{+}$and $F^{-}$are of rank at most $\leq 1$. Since they are symmetric and trace-free, they vanish identically.

Using again that $\left(e_{1} \cdot e_{3} \cdot \frac{\varphi^{--}}{\left|\varphi^{--}\right|}, e_{2} \cdot e_{3} \cdot \frac{\varphi^{--}}{\left|\varphi^{--}\right|}\right)$is an orthonormal frame of $\Sigma^{++}$, we have

$$
\nabla_{X} \varphi^{++}=F_{++}\left(X, e_{1}\right) e_{1} \cdot e_{3} \cdot \frac{\varphi^{--}}{\left|\varphi^{--}\right|}+F_{++}\left(X, e_{2}\right) e_{2} \cdot e_{3} \cdot \frac{\varphi^{--}}{\left|\varphi^{--}\right|} .
$$

Since $F_{++}=A_{++}-B_{++}$and denoting by $A^{++}$and $B^{++}$the operators of $T M$ associated to $A_{++}$and $B_{++}$and defined by

$A^{++}(X)=A_{++}\left(X, e_{1}\right) e_{1}+A_{++}\left(X, e_{2}\right) e_{2}, B^{++}(X)=B_{++}\left(X, e_{1}\right) e_{1}+B_{++}\left(X, e_{2}\right) e_{2}$, we get

$$
\nabla_{X} \varphi^{++}=\frac{1}{\left|\varphi^{--}\right|^{2}}\left[A^{++}(X) \cdot e_{3} \cdot \varphi^{--}-B^{++}(X) \cdot e_{3} \cdot \varphi^{--}\right]
$$

Similarly, we denote by $A^{--}$and $B^{--}$the operators of $T M$ associated to $A_{--}$and $B_{--}$. Thus, we have

$$
\nabla_{X} \varphi^{--}=\frac{1}{\left|\varphi^{++}\right|^{2}}\left[A^{--}(X) \cdot e_{3} \cdot \varphi^{++}-B^{--}(X) \cdot e_{3} \cdot \varphi^{++}\right] .
$$

Moreover, we easily get

and

$$
B^{++}(X) \cdot e_{3} \cdot \varphi^{--}=-\frac{1}{2}\left|\varphi^{--}\right|^{2}\left(\alpha(X+f X) \cdot \varphi^{-+}+\alpha h X \cdot \varphi^{+-}\right)
$$

Hence,

$$
B^{--}(X) \cdot e_{3} \cdot \varphi^{++}=-\frac{1}{2}\left|\varphi^{++}\right|^{2}\left(\alpha(X+f X) \cdot \varphi^{+-}+\alpha h X \cdot \varphi^{-+}\right) .
$$

$$
\begin{aligned}
\nabla_{X} \varphi^{+}= & \frac{1}{\left|\varphi^{--}\right|^{2}} A^{++}(X) \cdot e_{3} \cdot \varphi^{--}+\frac{\alpha}{2}(X+f X) \cdot \varphi^{-+}+\frac{\alpha}{2} h X \cdot \varphi^{+-} \\
& +\frac{1}{\left|\varphi^{++}\right|^{2}} A^{--}(X) \cdot e_{3} \cdot \varphi^{++}+\frac{\alpha}{2}(X+f X) \cdot \varphi^{+-}+\frac{\alpha}{2} h X \cdot \varphi^{-+} .
\end{aligned}
$$


Now, we set $A^{+}=A^{++}+A^{--}$. From the definition of $A^{++}$and $A^{--}$and since $F^{+}=0$, we have $\frac{A^{++}}{\left|\varphi^{--}\right|^{2}}=\frac{A^{--}}{\left|\varphi^{++}\right|^{2}}$. Bearing in mind that $\left|\varphi^{+}\right|^{2}=\left|\varphi^{++}\right|^{2}+\left|\varphi^{--}\right|^{2}$, we get finally

$$
\frac{A^{+}}{\left|\varphi^{+}\right|^{2}}=\frac{A^{++}}{\left|\varphi^{--}\right|^{2}}=\frac{A^{--}}{\left|\varphi^{++}\right|^{2}} \text {. }
$$

So, we have

$$
\nabla_{X} \varphi^{+}=\frac{1}{\left|\varphi^{+}\right|^{2}} A^{+}(X) \cdot e_{3} \cdot \varphi^{+}+\alpha(X+f X+h X) \cdot \varphi^{-} .
$$

Analogouslly, we set $A^{+-}$and $A^{-+}$the operators of $T M$ associated to $A_{+-}$and $A_{-+}$, and we denote $A^{-}=A^{+-}+A^{-+}$. Using the fact that $F^{-}=0$ we get

$$
\begin{aligned}
\nabla_{X} \varphi^{-}= & \frac{1}{\left|\varphi^{+-}\right|^{2}} A^{-+}(X) \cdot e_{3} \cdot \varphi^{+-}+\alpha(X+f X) \cdot \varphi^{++}+\alpha h X \cdot \varphi^{--} \\
& +\frac{1}{\left|\varphi^{-+}\right|^{2}} A^{+-}(X) \cdot e_{3} \cdot \varphi^{-+}+\frac{\alpha}{2}(X+f X) \cdot \varphi^{--}+\frac{\alpha}{2} h X \cdot \varphi^{++} \\
= & \frac{1}{\left|\varphi^{-}\right|^{2}} A^{-}(X) \cdot e_{3} \cdot \varphi^{-}+\frac{\alpha}{2}(X+f X+h X) \cdot \varphi^{+} .
\end{aligned}
$$

We now observe that formulas (31) and (32) also hold if $\varphi^{++}$or $\varphi^{--}$, (resp. $\varphi^{+-}$or $\varphi^{-+}$) vanishes at $p$ : indeed, assuming for instance that $\varphi^{++}(p)=0$, and thus that $\varphi^{--}(p) \neq 0$ since $\varphi^{+}(p) \neq 0$, equation (28) holds, and, from the norm condition in (25), we have

$$
\mathfrak{R} e\left\langle\nabla_{X} \varphi^{--}-\frac{\alpha}{2}(X+f X) \cdot \varphi^{+-}+\frac{\alpha}{2} h X \cdot \varphi^{-+}, \varphi^{--}\right\rangle=0 .
$$

Since $\left(\frac{\varphi^{--}}{\left|\varphi^{--}\right|}, i \frac{\varphi^{--}}{\left|\varphi^{--}\right|}\right)$is an orthonormal basis of $\Sigma^{--}$, we deduce that

$$
\nabla_{X} \varphi^{--}-\frac{\alpha}{2}(X+f X) \cdot \varphi^{+-}+\frac{\alpha}{2} h X \cdot \varphi^{-+}=i \delta(X) \frac{\varphi^{--}}{\left|\varphi^{--}\right|}
$$

for some real 1-form $\delta$. Moreover, since $\varphi^{++}=0$ at $p$, we have

$$
D \varphi^{--}+\alpha(2+\operatorname{tr}(f)) \varphi^{+-}+\alpha \beta \cdot \varphi^{-+}=0,
$$

which implies

$$
\left(\delta\left(e_{1}\right) e_{1}+\delta\left(e_{2}\right) e_{2}\right) \cdot \frac{\varphi^{--}}{\left|\varphi^{--}\right|}=0,
$$

and thus that $\delta=0$. We thus get $\nabla_{X} \varphi^{--}=\frac{\alpha}{2}(X+f X) \cdot \varphi^{+-}+\frac{\alpha}{2} h X \cdot \varphi^{-+}$instead of (29), which, together with (28), easily implies (31).

Now, we set

$$
\eta^{+}(X)=\left(\frac{1}{\left|\varphi^{+}\right|^{2}} A^{+}(X) \cdot e_{3}\right)^{+} \quad \text { and } \quad \eta^{-}(X)=\left(\frac{1}{\left|\varphi^{-}\right|^{2}} A^{-}(X) \cdot e_{3}\right)^{-}
$$

where, if $\sigma$ belongs to $\mathcal{C} l^{0}(T M \oplus E)$, we denote by $\sigma^{+}:=\frac{1+\omega_{4}}{2} \cdot \sigma$ and by $\sigma^{-}:=\frac{1-\omega_{4}}{2} \cdot \sigma$ the parts of $\sigma$ acting on $\Sigma^{+}$and on $\Sigma^{-}$only, i.e., such that

$$
\sigma^{+} \cdot \varphi=\sigma \cdot \varphi^{+} \in \Sigma^{+} \quad \text { and } \quad \sigma^{-} \cdot \varphi=\sigma \cdot \varphi^{-} \in \Sigma^{-} .
$$

Setting $\eta=\eta^{+}+\eta^{-}$we thus get

$$
\nabla_{X} \varphi=\eta(X) \cdot \varphi+\frac{\alpha}{2}(X+f X+h X) \cdot \varphi,
$$

as claimed in Proposition 4.1. 
Now, we will compute $B$ explicitely. For this, we set $A_{+}(X, Y):=\left\langle A^{+}(X), Y\right\rangle$ and $A_{-}(X, Y):=\left\langle A^{-}(X), Y\right\rangle$. Then, the form $\eta$ is given by

$$
\begin{aligned}
\eta(X)= & \frac{1}{2\left|\varphi^{+}\right|^{2}}\left[A_{+}\left(X, e_{1}\right)\left(e_{1} \cdot e_{3}-e_{2} \cdot e_{4}\right)+A_{+}\left(X, e_{2}\right)\left(e_{2} \cdot e_{3}+e_{1} \cdot e_{4}\right)\right] \\
& +\frac{1}{2\left|\varphi^{-}\right|^{2}}\left[A_{-}\left(X, e_{1}\right)\left(e_{1} \cdot e_{3}+e_{2} \cdot e_{4}\right)+A_{-}\left(X, e_{2}\right)\left(e_{2} \cdot e_{3}-e_{1} \cdot e_{4}\right)\right]
\end{aligned}
$$

with

$$
A_{+}(X, Y)=\mathfrak{R} e\left\langle\nabla_{X} \varphi^{+}-\frac{\alpha}{2}(X+f X+h X) \cdot \varphi^{-}, Y \cdot e_{3} \cdot \varphi^{+}\right\rangle
$$

and

$$
A_{-}(X, Y)=\mathfrak{R} e\left\langle\nabla_{X} \varphi^{-}-\frac{\alpha}{2}(X+f X+h X) \cdot \varphi^{+}, Y \cdot e_{3} \cdot \varphi^{-}\right\rangle .
$$

Moreover, we see easily by direct computations that for any vectors $X$ and $Y$ tangent to $M$,

$$
B(X, Y):=X \cdot \eta(Y)-\eta(Y) \cdot X
$$

is a vector belonging to $E$ which is such that

$$
\begin{aligned}
\langle B(X, Y), \xi\rangle= & \frac{1}{\left|\varphi^{+}\right|^{2}} \mathfrak{R} e\left\langle X \cdot \nabla_{Y} \varphi^{+}-\alpha(X+f X+h X) \cdot Y \cdot \varphi^{-}, \xi \cdot \varphi^{+}\right\rangle \\
& +\frac{1}{\left|\varphi^{-}\right|^{2}} \Re e\left\langle X \cdot \nabla_{Y} \varphi^{-}-\alpha(X+f X+h X) \cdot Y \cdot \varphi^{+}, \xi \cdot \varphi^{-}\right\rangle
\end{aligned}
$$

for all $\xi \in E$.

Lemma 5.6. The operator $B$ defined above is symmetric in $X$ and $Y$.

Proof: The proof is analogous to the symmetry of $A_{++}$proven above and uses the Dirac equations

and

$$
D \varphi^{+}=\vec{H} \cdot \varphi^{+}-\alpha\left[(2+\operatorname{tr}(f)) \varphi-^{-} \beta \cdot \varphi^{-}\right]
$$

$$
D \varphi^{-}=\vec{H} \cdot \varphi^{-}-\alpha\left[(2+\operatorname{tr}(f)) \varphi^{+}-\beta \cdot \varphi^{+}\right] .
$$

Now, computing

$$
\langle B(X, Y), \xi\rangle=\frac{1}{2}(\langle B(X, Y), \xi\rangle+\langle B(Y, X), \xi\rangle)
$$

we finally obtain that $B$ is given in the discussion of Section 4 .

Since $B\left(e_{j}, X\right)=e_{j} \cdot \eta(X)-\eta(X) \cdot e_{j}$, we obtain

$$
\sum_{j=1,2} e_{j} \cdot B\left(e_{j}, X\right)=-2 \eta(X)-\sum_{j=1,2} e_{j} \cdot \eta(X) \cdot e_{j} .
$$

Writing $\eta(X)$ in the form $\sum_{k=1,2} e_{k} \cdot \eta_{k}$ for some vectors $\eta_{k}$ belonging to $E$, we easily get that $\sum_{j=1,2} e_{j} \cdot \eta(X) \cdot e_{j}=0$. Indeed, we have

$$
\begin{aligned}
\sum_{j=1,2} e_{j} \cdot \eta(X) \cdot e_{j} & =\sum_{j=1,2} e_{j} \cdot\left(\sum_{k=1,2} e_{k} \cdot \eta_{k}\right) \cdot e_{j} \\
& =e_{1} \cdot\left(e_{1} \cdot \eta_{1}+e_{2} \cdot \eta_{2}\right) \cdot e_{1}+e_{2} \cdot\left(e_{1} \cdot \eta_{1}+e_{2} \cdot \eta_{2}\right) \cdot e_{2} \\
& =-\eta_{1} \cdot e_{1}-e_{2} \cdot \eta_{2}-e_{1} \cdot \eta_{1}-\eta_{2} \cdot e_{2} \\
& =e_{1} \cdot \eta_{1}+\eta_{2} \cdot e_{2}-e_{1} \cdot \eta_{1}-\eta_{2} \cdot e_{2} \\
& =0
\end{aligned}
$$


Thus, from (33), we get

$$
\eta(X)=-\frac{1}{2} \sum_{j=1,2} e_{j} \cdot B\left(e_{j}, X\right) .
$$

The last claim in Proposition 4.1 is now proved.

\section{REFERENCES}

[1] P. Bayard, On the spinorial representation of spacelike surfaces into 4-dimensional Minkowski space, J. Geom. Phys., in press.

[2] P. Bayard, M.A. Lawn \& J. Roth, Spinorial representation of surfaces in four-dimensional Space Forms, Ann. Glob. Anal. Geom., in press.

[3] C. Bär, Extrinsic bounds for eigenvalues of the Dirac operator, Ann. Glob. Anal. Geom., 16 (1998) 573-596.

[4] T. Friedrich, On the spinor representation of surfaces in Euclidean 3-space, J. Geom. Phys. (1998), 143-157.

[5] D. Kowalczyk, Isometric immersions into products of space forms, Geom. Dedicata 151 (2011), 1-8.

[6] M.A. Lawn \& J. Roth, Spinorial characterization of surfaces in pseudo-Riemannian space forms, Math. Phys. Anal. and Geom. 14 (2011) no. 3, 185-195.

[7] M.A. Lawn \& J. Roth, Isometric immersions of hypersurfaces into 4-dimensional manifolds via spinors, Diff. Geom. Appl. 28 (2) (2010), 205-219

[8] J.H. De Lira, R. Tojeiro \& F. Vitório, A Bonnet theorem for isometric immersions into products of space forms Arch. Math. (Basel) 95 (5) (2010), 469-479

[9] B. Morel, Surfaces in $\mathbb{S}^{3}$ and $\mathbb{H}^{3}$ via spinors, Actes du séminaire de théorie spectrale, Institut Fourier, Grenoble, 23 (2005), 9-22.

[10] R. Nakad \& J. Roth, Hypersurfaces of $\mathrm{Spin}^{\mathrm{c}}$ Manifolds and Lawson Correspondence, Ann. Glob Anal. Geom 42 (3) (2012), 421-442.

[11] P. Piccione and D.V. Tausk, An existence theorem for G-strcture preserving affine immersions, Indiana Univ. Math. J. 57 (3) (2008), 1431-1465.

[12] J. Roth, Spinorial characterization of surfaces into 3-dimensional homogeneous manifolds, J. Geom. Phys 60 (2010), 1045-106.

[13] J. Roth, Isometric immersion into Lorentzian products, Int. J. Geom. Method. Mod. Phys, 8 (2011) no.6, $1-22$.

(J. Roth) LAMA, Université Paris-Est Marne-la-Vallée, Cité Descartes, Champs sur Marne, 77454 MARNE-LA-VALLÉE CEDEX 2, FranCE

E-mail address: julien.rotheuniv-mlv.fr 